\title{
ENERGY EFFICIENT COOPERATIVE SPECTRUM SENSING IN COGNITIVE RADIO
}

\author{
Ramzi Saifan ${ }^{1}$, Ghazi Al-Sukar², Rawaa Al-Ameer ${ }^{2}$ and Iyad Jafar ${ }^{1}$ \\ ${ }^{1}$ Dept. Computer Engineering, \\ ${ }^{2}$ Dept. Electrical engineering \\ University of Jordan, Amman, Jordan 11942
}

\begin{abstract}
Sensing in cognitive radio $(C R)$ protects the primary user $(P U)$ from bad interference. Therefore, it is assumed to be a requirement. However, sensing has two main challenges; first the CR is required to sense the PU under very low signal to noise ratios which will take longer sensing time, and second, some CR nodes may suffer from deep fading and shadowing effects. Cooperative spectrum sensing (CSS) is supposed to solve these challenges. However, CSS adds extra energy consumption due to CRs send the sensing result to the fusion center and receive the final decision from the fusion center. This is in addition to the sensing energy itself. Therefore, CSS may consume considerable energy out of the battery of the CR node. Therefore in this paper, we try to find jointly the sensing time required from each $C R$ node and the number of $C R$ nodes who should perform sensing such that the energy and energy efficiency (i.e., ratio of throughput to energy consumed) are optimized. Simulation results show that the joint optimization achieves better in terms of energy efficiency than other approaches that perform separate optimization.
\end{abstract}

\section{KEYWORDS}

Cognitive Radio Network; Cooperative Spectrum Sensing; energy efficient sensing

\section{INTRODUCTION}

The concept of cognitive radio was first proposed by Joseph Mitola in a seminar at the Royal Institute of Technology in Stockholm in 1998 and published in an article by Mitola et al in 1999 [1]. It was a novel approach in wireless communications which may increase wireless spectrum utilization. It is based on using the channel of the licensed user (also called primary user, PU), if the PU is not using it and without harmful interference. The harmful interference is prevented by proper spectrum sensing.

Spectrum sensing is a continuous stage during the life cycle of cognitive radio communications where it must be done initially to find an idle channel and then must be done periodically to protect the PU, where if the PU is active again, sensing is used to find another idle channel. Therefore, reducing sensing time increases the room left for transmission which achieves the main target of $\mathrm{CR}$ of increasing wireless spectrum utilization. Moreover, sensing plays a crucial role in the success of the whole process, where the use of PU's channel is under the constraint that the PU is protected. PU protection is achieved by sensing. For this reason, sensing has received a lot of attention in order to perform it efficiently.

Sensing means detecting the existence of a primary transmitter on a specific channel. If the PU exists, then this channel cannot be used. Otherwise, the CRs are free to send on this channel. In primary transmitter detection, the $\mathrm{CR}$ is required to detect the existence of a primary transmitter in 
the keep-out region. The keep-out region can be imagined as a circle centered at the PU, and with a radius equal to the sum of the transmission range of the CR plus the transmission range of the PU. Therefore, PU sensing is under the challenge of detecting very weak signal with SNR typically down to $-20 \mathrm{db}$.

Another challenge for spectrum sensing is that it suffers from problems of multipath fading and shadowing which may decrease the detection performance. Therefore, cooperation is proposed in literature as a promising solution to these problems. It increases the probability of detection and decreases the probability of false alarm [2].

In CSS, there is a fusion center that collects the sensing results from different cognitive users and makes a decision about the presence or absence of a primary user.

Even though sensing in CR is one of the most studied areas in CR, research was focusing on either protecting the PU or reducing the sensing time such that the room left for transmission increases. In this paper, we consider sensing from another point of view which is reducing the consumed energy or increasing energy efficiency. Energy is consumed during sensing in three stages: 1) sensing stage itself consumes energy, where longer sensing time requires more energy. 2) Reporting stage, more cooperative nodes means more CR nodes are reporting to the fusion center and more reporting energy consumed. And 3) the sensing result affects transmission stage. If sensing result is true idle, then the CR node can send, and it consumes energy for transmission, but a good throughput is achieved. However, if the sensing result is false idle, then the node sends, which consumes energy, but with no good throughput since CR transmission interferes with the PU.

In this paper, we work on two targets which are reducing the sum of energy consumed in the sensing stages including sensing, reporting, and transmission. The second objective is maximizing energy efficiency which is the ratio of good throughput to the consumed energy. For that objective, we jointly find the sensing time required per CR node and the number of nodes who should perform cooperative sensing.

State-of-the-art papers sometimes assume a fixed sensing time and a variable reporting time [3]. Others assumed fixed transmission time and variable sensing and reporting times. In this paper, we assume variable sensing, reporting and transmission times. This is supposed to give better performance since we increase the degrees of freedom.

Although the work in [5] has some similarities to ours, there are two main differences between both works:

- Different time distribution mechanisms are assumed, in [5] the transmission time is fixed, while we don't fix the transmission time.

- We propose energy efficiency as an objective function to be maximized by jointly finding the sensing time and number of cooperating CRs who perform spectrum sensing. While the optimization problem in [5] solves only one parameter which is the number of cognitive users that maximizes the energy efficiency.

The rest of the paper is organized as follows: in Section 2 we show some related work. In Section 3 , we introduce problem definition and system model. Performance measures and the optimization problem are in Sections 4 and 5 respectively. Simulation results are in Section 6. Finally, we conclude in Section 7. 


\section{RELATED WORK}

Many techniques have been proposed in the literature to improve energy efficiency of cooperative spectrum sensing. We can classify the techniques according to spectrum sensing stage optimization into three types: 1) Energy efficient local sensing, 2) energy efficient reporting, and 3) energy efficient decision

Number of sensing users and the sensing time are significant parameters of the local sensing energy consumption. Therefore, decreasing energy consumption in the sensing stage can be achieved in two different techniques, either reducing number of sensing users or by reducing the sensing time.

The authors in [3] formulated the minimum number of sensing CRs that satisfies predefined constraints on detection and false-alarm probabilities. They studied only the energy consumed in sensing stage, and did not consider energy consumed in reporting and data transmission stages. In [4], the authors reduced sensing energy consumption by dividing the cognitive users into several subsets and only one subset at certain period is activated.

The optimal number of sensing users is computed iteratively in [5] while maximizing energy efficiency. The analysis is based on limited time resources assumption and fixed transmission time. This implies that the time resources dedicated for CSS process are limited and shared between spectrum sensing and results reporting.

Optimizing sensing time plays an important factor in the energy consumed in cooperative spectrum sensing. The authors of [6] investigated sensing time for individual sensing systems. They presented an adaptive sensing time based on the past spectrum occupancy pattern.

The CRs perform coarse sensing that is an initial short sensing stage in [7]. If the sensing result stays in the predefined range, the CR does not report the local decision to the fusion center; otherwise, the CR reports the local decision to the fusion center. In the first case, fusion center makes a global decision only if the majority decides it. If there is no majority, then all CRS use another sensing stage that is called fine sensing. The fine sensing stage is two times longer than that of the coarse stage. This two-stages sensing scheme can decrease the sensing time but it needs extra energy consumption in reporting stage, this is because it is repeated twice and also because of the waiting of the first global decision. This will most likely affect energy efficiency.

Reporting stage also consumes significant amount of energy out of the energy consumed in the whole sensing process. In 2011, [3] optimized censoring thresholds to decrease the energy consumption. Censoring is a technique that can reduce the reporting CRs. In this strategy, if a CR lies inside a specific range, it reports its sensing result. Lee and Wolf in [8] introduced a confidence-voting scheme, where each CR sends its sensing result only if it has a given confidence level which is computed from the history of the local result compared to the final result. All CRs still sense the spectrum, which consumes large amount of energy. In [9], the authors proposed an alternative approach which is called cluster-based spectrum sensing. It foresees that each group of CRs selects a cluster-head to process its results and to report just one decision on behalf of all of the group members. Also in this case, all users still sense the spectrum. In addition, extra energy is consumed due to the information exchange between the cluster-head and cluster-members. 


\section{System Model And Problem Definition}

In this paper, we introduce an algorithm to reduce the consumed energy, while keeping the detection accuracy over the desirable bound. We investigate the problem of optimizing the number of sensing users and the sensing time of each CR node for CSS by energy efficiency maximization while satisfying a predefined constraint on the detection probability.

We consider a cognitive radio system that consists of a fusion center and $\mathrm{N}_{\mathrm{T}} \mathrm{CRs}$. The CRs work to detect the activity of a primary user on a given spectrum band, as shown in Figure 1. Each working CR receives the primary signal with signal to noise ratio (SNR). We assume that the distances between the cognitive users are small compared with the distance to the primary transmitter. Therefore, it is assumed that each channel gain has the same variance. Also the average received SNR is the same at each CR [5].

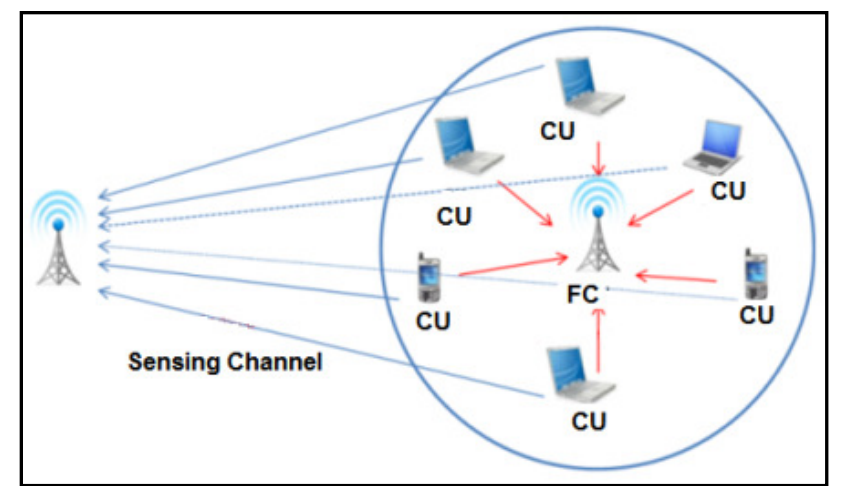

Figure 1. Cooperative spectrum sensing system.

Each of the cooperative CRs is supposed to employ an energy detector and measures the received power on the channel during the sensing period. In energy detection, each CR collects energy samples, where the signal observed $y(t)$ by each CR is as in Equation 1.

$$
y(t)= \begin{cases}x(t)+n(t) & \text { if the channel is busy, } H_{1} \\ n(t) & \text { if the channel is free, } H_{0}\end{cases}
$$

Where $n(t)$ is additive white Gaussian noise with variance $\sigma_{\mathrm{n}}^{2} . x(t)$ is the received primary user signal which is assumed to be Circularly Symmetric Complex Gaussian (CSCG) distributed with variance $\sigma_{\mathrm{x}}^{2}$.

After local sensing, the final decision of spectrum status is cooperatively made by reporting the local decisions to the Fusion Center (FC). We used Time Division Multiple Access Scheme (TDMA) for reporting the local decisions to the FC. We chose the soft scheme: each cognitive user sends the average of the energy detector to the FC. At the FC, the average of all received data from the cognitive users $(A v)$ is compared with a predefined threshold $(\lambda)$, and a decision is made between $H O$ (i.e., primary user is absent) and $H I$ (i.e., primary user is present) as follows:

$$
\text { Decision }=\left\{\begin{array}{lll}
H 1 & (\text { busy }), & A v \geq \lambda \\
H 0 & (\text { free }), & A v<\lambda
\end{array}\right.
$$


We assume that each CR node is provided with a single transceiver. Therefore, the CR cannot transmit and sense at the same time. Consequently, CR needs periodic spectrum sensing, Figure 2 shows the frame structure. Frame duration (T) is divided into three slots, these are: sensing, reporting and transmission.

1. Sensing slot $(T s)$ : when $N$ CRs sense the spectrum and collect energy samples. At sensing time, none of the $N_{T}$ CRs is allowed to transmit on the same channel because we assume energy detection where any measured energy will be considered as PU signal. Note that $N$ can be $\leq N_{T}$.

2. Reporting slot $(T r)$ : after the CRs make local measurements, they forward them to the fusion center. The CRs cannot report at the same time over one channel because the receiver is common. Thus, we consider consecutive reporting based on TDMA scheme, where each user has its own reporting time slot.

3. Transmission slot $(T t)$ : if the channel is decided to be unused, one or multiple CRs are scheduled for data transmission.

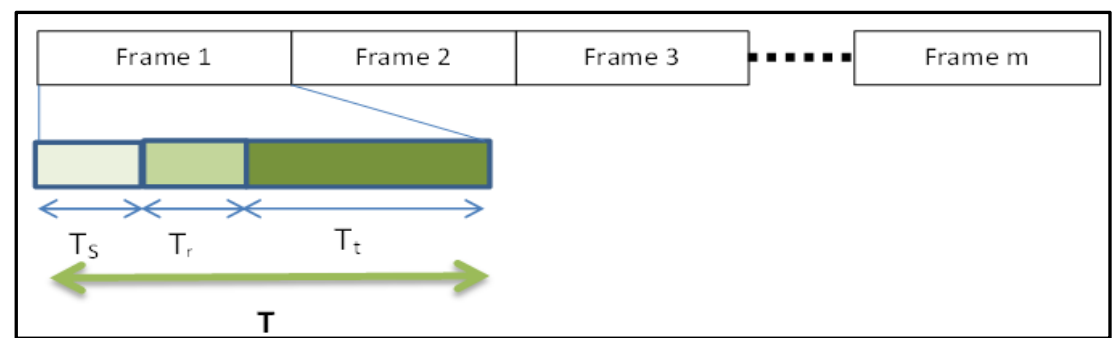

Figure 2. Frame structure for cooperative spectrum sensing in CR.

As a consequence:

$$
T=T_{S}+T_{r}+T_{t}
$$

Althunibat, et al [5] solved an optimization problem based on a limited time resources constraint. More specifically, they assumed that the total frame has a finite and fixed duration. A fixed part of it is dedicated for data transmission, while the rest is distributed between sensing time and reporting time as a function of the number of sensing users. With this finite frame duration assumption, if the number of users increases, the reporting time has to be longer, and thus, a shorter time is left for local sensing.

If we consider $\tau$ as the time needed by each CR to report the sensed result to the FC, then the total reporting time for all sensing CRs is $T_{r}=N^{*} \tau$. The duration of the sensing can be expressed as a function of number of CRs:

$$
T_{S}(N)=T-T_{t}-\mathrm{N} * \tau
$$

The number of collected samples per user as a function of the number of sensing users is:

$$
S(N)=\left(T-T_{t}-N * \tau\right) * f_{S}
$$

Where: $f_{s}$ is the sampling frequency 


\section{Performance Metrics Of CoOperative Spectrum Sensing}

Overall cooperative spectrum sensing performance is evaluated by the detection accuracy of the global decision. A combination of the detection probability and false alarm probability represents the detection accuracy. On the other side, the achievable throughput and total energy consumption represent important metrics in any communication network.

\subsection{Detection Accuracy}

The detection probability $\left(\mathrm{P}_{\mathrm{D}}\right)$ is the probability that the fusion center identifies a busy channel as busy. Suppose that SNR is the signal-to-noise ratio $=\left(\frac{\sigma_{x}^{2}}{\sigma_{n}^{2}}\right)$, then:

$$
P_{D}=P(H 1 / H 1)=P(S N R \geq \lambda / H 1)
$$

As in [5], $\mathrm{P}_{\mathrm{D}}$ is given as follows:

$$
P_{D}=Q\left(\frac{\lambda-(1+S N R)}{\frac{(1+S N R)}{\sqrt{N * S}}}\right)
$$

Where: $Q(x)=\frac{1}{\sqrt{2 \pi}} \int_{x}^{\infty} \exp \left(\frac{-t^{2}}{2}\right) d t$

$\mathrm{N}$ is the actual number of sensing CRs with $1 \leq \mathrm{N} \leq \mathrm{N}_{\mathrm{T}}$, and $\mathrm{S}$ is the number of energy samples by each CR.

The false alarm probability $\left(\mathrm{P}_{\mathrm{F}}\right)$ is the probability that the FC identifies an unused channel as busy, $\mathrm{P}_{\mathrm{F}}$ is given as follows:

$$
P_{F}=P(H 1 / H 0)=P(S N R \geq \lambda / H 0)
$$

Also, according to [5], $\mathrm{P}_{\mathrm{F}}$ is given as follows:

$$
P_{F}=Q\left(Q^{-1}\left(P_{D}^{t h}\right) *(1+S N R)+S N R * \sqrt{N * S}\right)
$$

It is assumed that the chosen threshold (i.e., $\lambda$ ) guarantees a given detection probability $\left(P_{D}^{t h}\right)$. The constraint $P_{D}^{t h}$ is used to limit the interference at the primary user caused by miss detection.

\subsection{Energy Consumption}

The consumed energy in cooperative spectrum sensing by all CRs is related to the sum of three components:

1) Energy consumed during local sensing $\left(E_{s}\right)$.

$$
E_{S}(N)=N * T_{S}(N) * \rho_{S}
$$


2) Energy consumed during result reporting $\left(E_{r}\right)$.

$$
E_{r}(N)=N * \tau * \rho_{r}
$$

3) Energy consumed during data transmission $\left(E_{t}\right)$.

$$
E_{t}(N)=T_{t} * \rho_{t}=(\mathrm{T}-\mathrm{TS}(\mathrm{N})-\mathrm{N} * \tau) * \rho_{t}
$$

where $\rho_{S}, \rho_{r}$ and $\rho_{t}$ are the consumed powers per CR for local sensing, reporting, and data transmission, respectively.

Probability of transmitting data is the same as the probability of identifying the spectrum as free during cooperative spectrum sensing:

$$
P_{\text {free }}=1-P_{1} * P_{D}-P_{0} * P_{F}
$$

Where

$P_{0}$ : The probability that the spectrum is unused

$P_{1}$ : The probability that the spectrum is used

The energy consumed as a function of $N$ is given as follows:

$$
E(N)=E_{S}(N)+E_{r}(N)+P_{\text {free }} * E_{t}
$$

It is worth mentioning that increasing $N$ does not necessarily increase the total energy consumption. This is due to the contradicting effects on $E_{s}, E_{r}$ and $P_{\text {free }}$.

\subsection{Achievable Throughput}

The achievable throughput of CSS can be defined as the average amount of the successfullydelivered transmitted bits. A successful transmission occurs only in the case of correct identification of the unused spectrum. In other words, the transmitted bits are successfully delivered if the channel was unused and correctly identified as free. Hence, the achievable throughput (Th), measured in bits/s, is given as:

$$
T h=P_{0} *\left(1-P_{F}\right) * D_{t} * T_{t}
$$

Where the factor $P_{0} *\left(1-P_{F}(N)\right)$ represents the probability of correct identification of the unused spectrum, and $D_{t}$ is the data transmission rate in bits/s.

\subsection{Energy Efficiency}

Finally, the energy efficiency is defined as the average throughput divided by the average consumed energy as follows:

$$
\mu(N)=\frac{P_{0} *\left(1-P_{F}\right) * D_{t} * T_{t}}{E_{S}(N)+E_{r}(N)+P_{\text {free }} * E_{t}(N)}
$$




\section{JOINT OPTIMIZATION ENERgY EFFICIENT ALgORITHM}

The number of sensing CRs and sensing time are significant parameters of local sensing energy consumption. For this reason, improving energy efficiency can be achieved in two different techniques: by reducing the number of sensing users and optimizing the sensing time. In this section, we propose Joint Optimization Energy Efficient Algorithm to choose the optimal number of sensing CRs and their optimal sensing. We assume that the total frame has a finite and fixed duration while sensing, reporting and transmission times are variable.

\subsection{Energy Consumption Minimization}

There is a tradeoff between the selected $N$ and $T_{s}$ from one side and the consumed energy from the other side. If $N$ increases, $P_{F}$ decreases, as indicated in Equation (9), which increases the energy consumed during transmission, as indicated in Equations (13) and (14), but the good throughput increases also. However, larger $N$ implies more reporting energy and less transmission time according to Equations 11 and 12 which decreases good throughput.

Also, the same equations indicate that if $T_{s}$ increases, number of samples increases, $\mathrm{P}_{\mathrm{F}}$ decreases, which increases the probability of transmitting data and consumed energy during the transmission time, as shown by Equation (12). But also, good throughput increases. However, as $T_{s}$ decreases, $P_{F}$ decreases, which increases transmission energy without good throughput. Therefore, selected values of $N$ and $T_{s}$ affect energy consumption.

\subsection{Average Throughput Maximization}

There is also a tradeoff between the selected $N$ and $T_{s}$ from one side and the achieved throughput from the other side. Increasing the number of sensing users leads to a higher accuracy in the received sensing results, which improves the achievable throughput. On the other hand, larger number of sensing users consumes more time in reporting process. This will decrease room left for transmission and as a result throughput is decreased as indicated in Equation (15). Also, increasing the sensing time for each user leads to increase the number of energy samples which improves the accuracy of sensing results. On the other hand, more energy samples will decrease the transmission time, which decreases throughput, Equation (15). Therefore, it is necessary to select $T_{s}$ and $N$ so that, the throughput is maximized.

\subsection{Energy Efficiency Maximization}

Energy efficiency is defined as the ratio of throughput to energy consumption. Therefore, maximizing it achieves the balance point between energy consumption and average throughput. Energy efficiency, as defined in Equation (16) can be maximized as follows:

$$
\max \mu(N, T s)=\max _{\left(\mathrm{N}, \mathrm{T}_{\mathrm{S}}\right)} \frac{P_{0} *\left(1-P_{F}\right) * D_{t} * T_{t}}{E_{S}(N)+E_{r}(N)+P_{\text {free }} * E_{t}(N)}
$$

We are going to find $N$ and $T_{S}$ which maximize the energy efficiency. To solve this maximization, we will use the bisection algorithm. The procedure of the proposed algorithm is illustrated using the pseudo code shown in Figure 3, where $\mu$ refers to the energy efficiency, $N_{O P T}$ is the optimal number of sensing CRs, and Tsopt is the optimal sensing time for these sensing CRs. $N_{\min }, N_{\max }$, $T_{s-\min }$ and $T_{\text {smax }}$ are the boundary values of number of sensing users and sensing time respectively. 


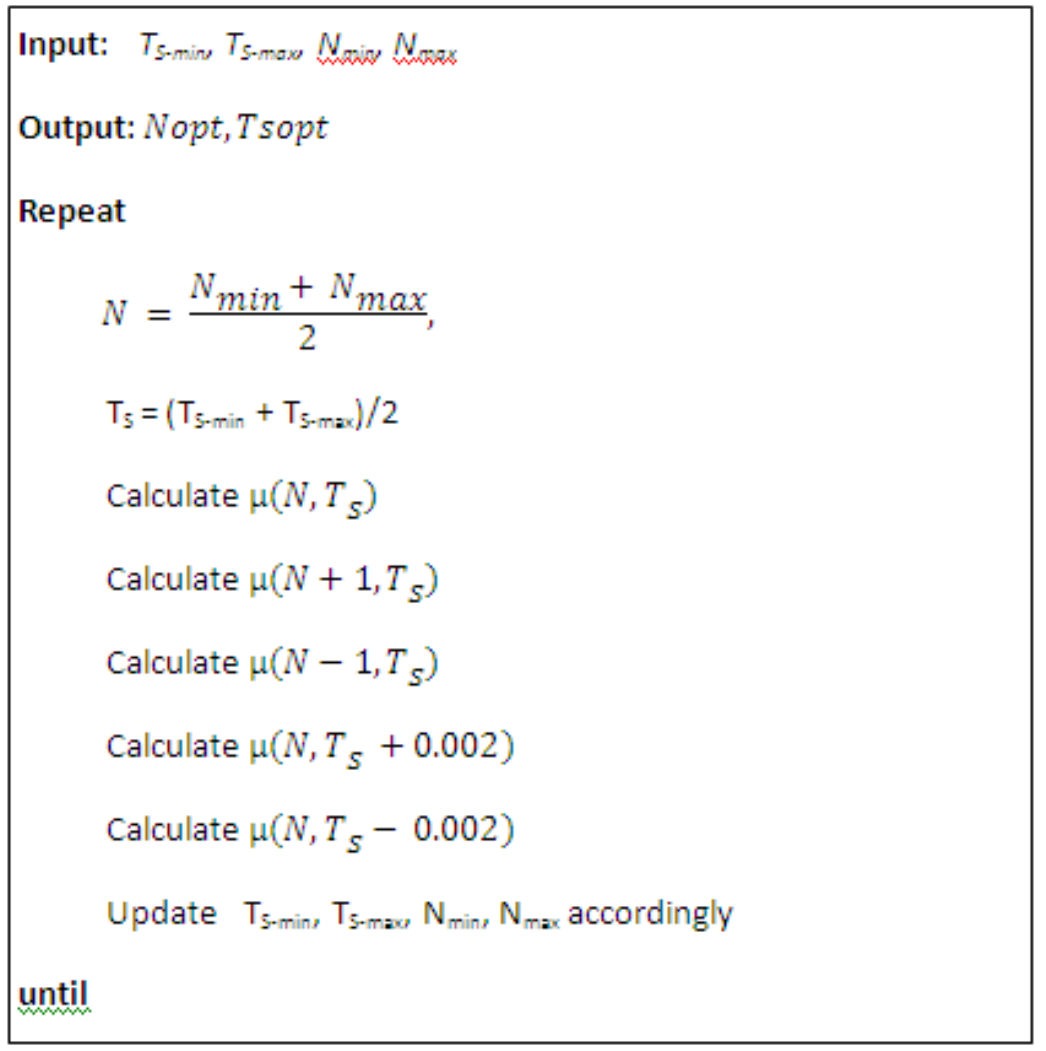

Figure 3: Pseudo code of proposed algorithm

Table 1: Simulation parameters

\begin{tabular}{|l|l|}
\hline Parameter & value \\
\hline Probability of idle $(\mathrm{P} 0)$ & 0.5 \\
\hline Sampling Frequency $(\mathrm{fs})$ & $1 \mathrm{MHz}$ \\
\hline Sensing power Consumption $\left(\boldsymbol{\rho}_{\mathbf{S}}\right)$ & $0.1 \mathrm{~W}$ \\
\hline Reporting Power Consumption $\left(\boldsymbol{\rho}_{\mathbf{r}}\right)$ & $1 \mathrm{~W}$ \\
\hline Transmission Power Consumption $\left(\boldsymbol{\rho}_{\mathbf{t}}\right)$ & $1 \mathrm{~W}$ \\
\hline Frame Length $(\mathrm{T})$ & $100 \mathrm{~ms}$ \\
\hline Data Rate $(\mathrm{Dt})$ & $200 \mathrm{Kbps}$ \\
\hline SNR & $-20 \mathrm{~dB}$ \\
\hline
\end{tabular}

\section{SIMULATION RESULTS}

Simulation parameters are listed in Table 1. Average throughput versus the number of sensing users is presented in Figure 4, for multiple values of sensing time at a fixed detection probability $\mathrm{P}_{\mathrm{D}}=0.9$. Note that in the first part of each curve, as $\mathrm{N}$ increases, the number of collected energy samples increases because number of sensing users is increased. More energy samples means longer sensing time, which leads to $P_{F}$ decrease and increase in the probability of transmitting data, which in turn increases the throughput. However, the throughput curves will not keep increasing. They will decrease again because as number of sensing CRs increases, reporting time $\left(T_{r}\right.$ increases. Since the frame structure is limited, when $T_{r}$ increases, $T_{t}$ decreases, 
and therefore the throughput decreases. It is necessary to optimize the number of sensing users. Note that the maximum average throughput occurs at $N=23$ when $T s=1 \mathrm{~ms}$. However, the maximum average throughput is dependent on multiple factors as shown in the figure which is dependent Ts, $\mathrm{N}$, and other simulation parameters. Therefore, the optimal point is different and requires optimization.

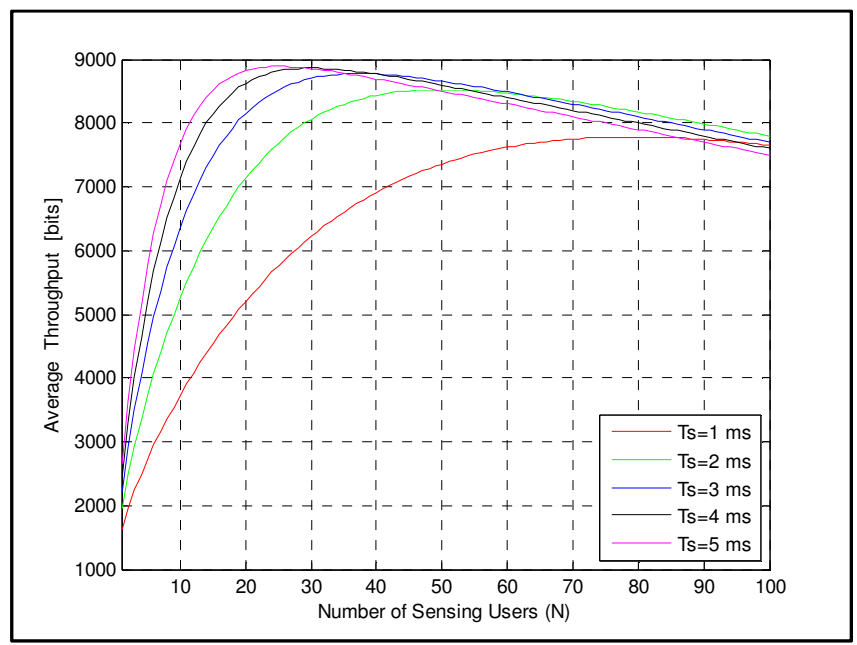

Figure 4. Achievable throughput versus number of sensing nodes

Average throughput versus sensing time is presented in Figure 5. Note that increasing the sensing time for each CR leads to increase in the number of samples which improves the accuracy (smaller false alarm and miss-detection probabilities) of sensing results. Smaller false alarm probability increases the chances of transmission and hence increases the throughput. Additionally, smaller miss-detection probability reduces un-useful transmission cases in which the CR node interferes with the PU transmission. On the other hand, more sensing time leads to decrease in transmission time, which decreases throughput. Therefore, curves have peaks where they start increasing (due to more accurate sensing) then decreasing again (due to less transmission time).

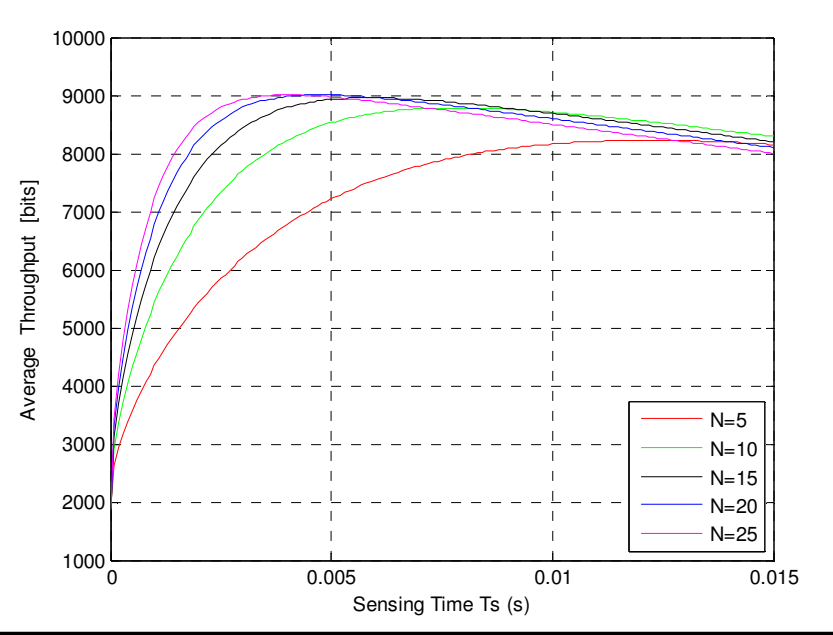

Figure 5. Achievable throughput versus sensing time 
Figure 6 shows the energy efficiency versus the number of sensing users presented for multiple values of $T s$. Figure 7 shows the energy efficiency versus the sensing time presented for multiple values of $N$. Notice that the maximum energy efficiency is achieved at a low value of $N$, and it increases as $T s$ increases; we can clearly note that there is an optimal sensing time and optimal number of sensing CRs for each number of available CRs. Therefore, the joint optimization of the sensing time and the number of sensing CRs is important for cognitive network to improve energy efficiency.

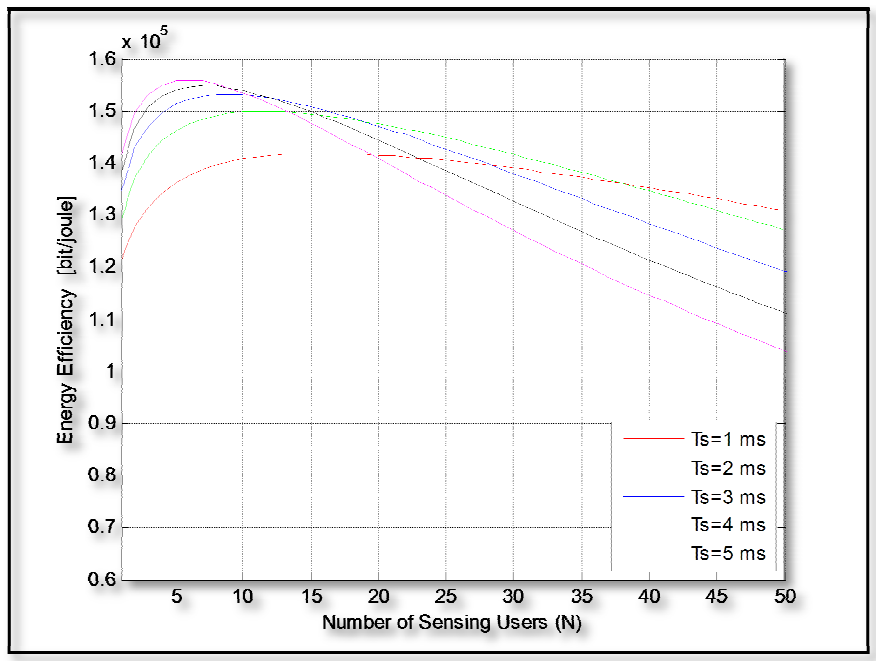

Figure 6. Energy efficiency performance versus number of sensing nodes

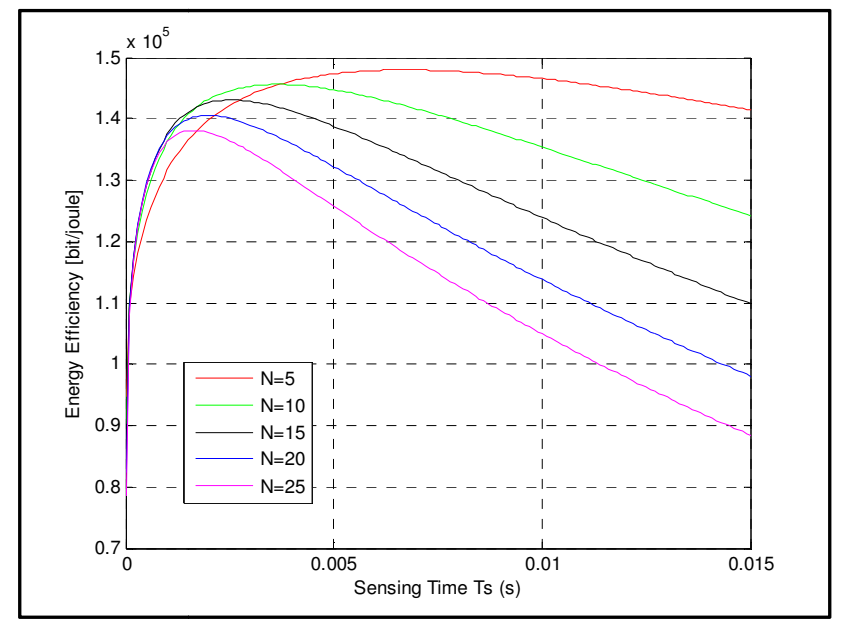

Figure 7. Energy efficiency performance versus different sensing times.

Fig. 8 shows energy efficiency versus number of sensing CRs at a fixed detection probability $\mathrm{P}_{\mathrm{D}}=0.9$. The joint optimization energy efficient algorithm achieves the best energy efficiency. Althunibat algorithm [5] and the case without optimization approach are identical when there are 1 to 7 cognitive users, our proposed algorithm gives better results than [5] because we had optimized the sensing time along with the number of sensing CRs, while [5] algorithm only optimizes number of sensing CRs. 


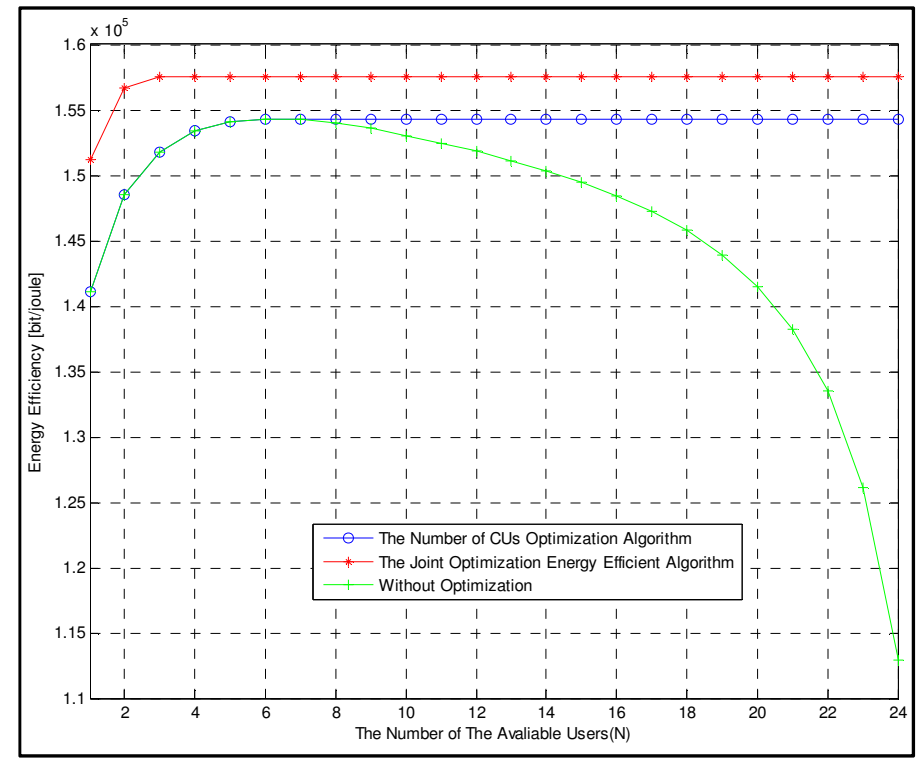

Figure 8. Energy Efficiency versus the number of available users.

\section{CONCLuSion}

In this paper, we worked on cooperative spectrum sensing. We assumed slotted time. Each time slot composed of sensing, reporting and transmission sub-slots. All of the three sub-slots are assumed to be variable, and they are supposed to be function of number of cooperative sensing CRs. We showed by simulation that there is an optimal sensing time which maximizes energy efficiency. Also, there is an optimal number of cooperating nodes that maximizes energy efficiency. Therefore, we find jointly the number of cooperating CRs and the sensing time of the CR nodes which maximize energy efficiency.

\section{REFERENCES}

[1] Mitola, M. Maguire, Jr. (1 999), Cognitive Radio: Making Software Radios More Personal, IEEE Pcrsonal Communications, Auguat volume pp.13-18.

[2] Yucek, T. and Arslan, H. (2009), A Survey of Spectrum Sensing Algorithms for Cognitive Radio Applications. IEEE Communications Surveys \& Tutorials, VOL. 11, NO. 1, first quarter, pp.116-130.

[3] Maleki, S. Pandharipande, A. and Leus, G. (2011) Energy-Efficient Distributed Spectrum Sensing for Cognitive Sensor Networks. IEEE Sensors Journal, VOL. 11, NO. 3, pp.565 -574.

[4] Deng, R. Chen, J. Yuen, C. Cheng, P. and Sun, Y. (2012), Energy-Efficient Cooperative Spectrum Sensing by Optimal Scheduling in Sensor-Aided Cognitive Radio Networks, IEEE Transactions On Vehicular Technology, VOL. 61, NO. 2.

[5] Althunibat, S. Renzo, M.D. and GranelliM, F. (2014), Cooperative spectrum sensing for cognitive radio networks under limited time constraints. Elsevier Journals, Computer Communications 43, pp.55-63.

[6] Su, H. and Zhang, X. (2010),Energy-Efficient Spectrum Sensing for Cognitive Radio Networks, IEEE ICC 2010 Conference.

[7] Zhao, Nan, et al. "Energy-efficient cooperative spectrum sensing schemes for cognitive radio networks." EURASIP Journal on Wireless Communications and Networking 2013.1 (2013): 1-13.

[8] Lee, C and Wolf, W. (2008), Multiple access-inspired cooperative spectrum sensing for cognitive radio. ieee communications letters.

[9] Sun, Chunhua, Wei Zhang, and K. Ben. "Cluster-based cooperative spectrum sensing in cognitive radio systems." Communications, 2007. ICC'07. IEEE International Conference on. IEEE, 2007. 